Wright State University

CORE Scholar

Mathematics and Statistics Faculty

Publications

Mathematics and Statistics

1985

\title{
Cramer Type Large Deviations for Generalized Rank Statistics
}

Munsup Seoh

Wright State University - Main Campus, munsup.seoh@wright.edu

Stegan S. Ralescu

Madan L. Puri

Follow this and additional works at: https://corescholar.libraries.wright.edu/math

Part of the Applied Mathematics Commons, Applied Statistics Commons, and the Mathematics Commons

\section{Repository Citation}

Seoh, M., Ralescu, S. S., \& Puri, M. L. (1985). Cramer Type Large Deviations for Generalized Rank Statistics. Annals of Probability, 13 (1), 115-125.

https://corescholar.libraries.wright.edu/math/30

This Article is brought to you for free and open access by the Mathematics and Statistics department at CORE Scholar. It has been accepted for inclusion in Mathematics and Statistics Faculty Publications by an authorized administrator of CORE Scholar. For more information, please contact library-corescholar@wright.edu. 


\title{
CRAMÉR TYPE LARGE DEVIATIONS FOR GENERALIZED RANK STATISTICS ${ }^{1}$
}

\author{
By Munsup Seoh, Stefan S. Ralescu and Madan L. Puri \\ Wright State University, Brown University, and Indiana University
}

A Cramér type large deviation theorem is proved under alternatives as well as under hypothesis for the generalized linear rank statistic which includes as special cases (unsigned) linear rank statistics, signed linear rank statistics, linear combination of functions of order statistics, and a rank combinatorial statistic.

1. Introduction. Let $X_{N 1}, X_{N 2}, \cdots, X_{N N}$ be independent r.v.'s (random variables) and let $g$ be a real valued measurable function such that $X_{N j}^{g} \equiv g\left(X_{N j}\right)$, $1 \leq j \leq N$, has a c.d.f. (cumulative distribution function) $G_{N j}$. We introduce the generalized linear rank statistic

$$
T_{N}=\sum_{j=1}^{N} c_{N j} a_{N R_{N j}^{k}}\left(X_{N j}\right)
$$

where $\left\{c_{N j}: 1 \leq j \leq N\right\}$ is an array of regression constants; $\left\{a_{N j}(\cdot): 1 \leq j \leq N\right\}$ is an array of known real functions (called scores); and $R_{N j}^{g}=\sum_{k=1}^{N} u\left(X_{N j}^{g}-X_{N k}^{g}\right)$ is a generalized rank of $X_{N j}^{g}$ among $\left\{X_{N k}^{g}: 1 \leq k \leq N\right\}$ where $u(x)=1$ if $x \geq 0$ and $u(x)=0$ otherwise. We assume that the scores $a_{N j}(\cdot), 1 \leq j \leq N$ are generated by a nonconstant score generating function $\phi(s, t), 0<s<1,-\infty<t$ $<\infty$, in either of the following two ways;

$$
\begin{aligned}
& a_{N j}(t)=\phi\left(E U_{N: j}, t\right), \quad j=1,2, \cdots, N \text { (approximate scores) } \\
& a_{N j}(t)=E \phi\left(U_{N: j}, t\right) \quad j=1,2, \cdots, N \text { (exact scores) }
\end{aligned}
$$

where $U_{N: j}$ is the $j$ th order statistic in a random sample of size $N$ from the uniform distribution over $(0,1)$. To avoid the trivialities, we assume that $\sum_{j=1}^{N}\left|c_{N j}\right|>0$.

When $g(x)=x$ and $\phi(x, y)=\tilde{\phi}(x)$, the statistic (1.1) reduces to the (unsigned) linear rank statistic

$$
T_{N}=\sum_{j=1}^{N} c_{N j} \tilde{a}_{N R_{N j}}
$$

where $R_{N j}, 1 \leq j \leq N$, is the rank of $X_{N j}$ among $\left\{X_{N k}: 1 \leq k \leq N\right\}$ and $\tilde{a}_{N j}$ 's are usual scores of constants. On the other hand, when $g(x)=|x|$ and $\phi(x, y)=$ $\tilde{\phi}(x) \operatorname{sgn} y$, where sgn $y=2 u(y)-1$, the statistic (1.1) reduces to the signed linear

Received July 1983; revised March 1984.

Research supported by NSF Grant MCS-8301409.

AMS 1980 subject classifications. Primary 60F10; secondary 62E20.

Key words and phrases. Linear rank statistics, order statistics, rank combinatorial statistic, large deviation probabilities. 
rank statistic

$$
T_{N}=\sum_{j=1}^{N} c_{N j} \tilde{a}_{N R_{N j}^{+}} \operatorname{sgn} X_{N j}
$$

where $R_{N j}^{+}, 1 \leq j \leq N$, is the rank of $\left|X_{N j}\right|$ among $\left\{\left|X_{N k}\right|: 1 \leq k \leq N\right\}$.

Also note that when $c_{N 1}=\ldots=c_{N N}=N^{-1}, g(x)=x, \phi(x, y)=\tilde{\phi}(x) \psi(y)$, and the underlying c.d.f.'s $G_{N j}$ are continuous, the statistic (1.1) reduces to the linear combination of functions of order statistics

$$
T_{N}=(1 / N) \sum_{j=1}^{N} \tilde{a}_{N R_{N j}} \psi\left(X_{N j}\right)=(1 / N) \sum_{j=1}^{N} \tilde{a}_{N j} \psi\left(X_{N: j}\right)
$$

where $X_{N: j}$ is the $j$ th order statistic among $\left\{X_{N k}, 1 \leq k \leq N\right\}$. Furthermore, denoting $Y_{j k}=c_{N j} a_{N k}\left(X_{N j}\right), 1 \leq j, k \leq N$, we can rewrite (1.1) as

$$
T_{N}=\sum_{j=1}^{N} Y_{j R_{N j}^{g}}
$$

which is a rank combinatorial statistic. For these four different types of statistics, several authors have investigated problems concerning asymptotic normality, rate of convergence to normality and higher order expansions. For a review, the reader is referred to Hájek $(1962,1968)$, Hušková $(1970,1977,1979)$, Jurečková and Puri (1975), Bergström and Puri (1977), Puri and Seoh (1984a, b, c), Does (1982), Puri and Wu (1983) (for statistics of the type (1.4) and (1.5)); Shorack (1969, 1972), Stigler (1974), Bjerve (1977) and Helmers (1977, 1980, 1981) (for statistics of the type (1.6)); Hoeffding (1951), Motoo (1956), von Bahr (1976) and Ho and Chen (1978) (for the statistics of the type (1.7)) and the papers cited therein, among others.

In recent years, there has been a great upsurge of activity in the theory of large deviations initiated by Cramér (1938) and studied in detail by Petrov (1975) for the case of independent summands. Large deviation probabilities for $U$-statistics were obtained by Malevich and Abdalimov (1979) while the corresponding results for the case of the statistic (1.4) were studied by Kallenberg (1982). But these results are restrictive in the sense that the observations $X_{N 1}, X_{N 2}$, $\cdots, X_{N N}$ are identically distributed and the underlying distribution function is continuous (Kallenberg, 1982). In addition, Robinson (1977) has dealt with the case of large deviations for samples from finite populations.

In this paper we shall be concerned with the relative error of the normal approximation to the distribution of the (properly normalized) generalized linear rank statistic (1.1) under general alternatives, i.e., assuming only that the observations are independent (not necessarily identically distributed) and without assuming the continuity of the underlying distribution functions. The results obtained not only include the results of Kallenberg (1982) as a special case, but also provide the large deviation theory for the statistics of the type (1.5), (1.6), and (1.7) which to the best of our knowledge has not been considered in the generality of the present paper. 
2. Assumptions and main theorem. Throughout this paper we make the following assumptions.

Assumption (A). The variance of $T_{N}$ satisfy

$$
\lim \inf \tau_{N}^{2}=\lim \inf \operatorname{Var} T_{N}>0
$$

Assumption (B). The regression constants $c_{N 1}, c_{N 2}, \cdots, c_{N N}$ satisfy

$$
\sum_{j=1}^{N} c_{N j}^{2}=1, \quad \max _{1 \leq j \leq N}\left|c_{N j}\right| \leq A_{1} N^{-1 / 3}, \quad \sum_{j=1}^{N}\left|c_{N j}\right|^{3} \leq A_{2} N^{-1 / 2},
$$

where $A_{1}$ and $A_{2}$ are absolute constants.

Assumption (C). The score generating function $\phi(s, t)$ is differentiable with respect to its first argument $s$ such that its first partial derivatives $\phi_{1}(s, t)=$ $\partial \phi(s, t) / \partial s$ satisfy Lipschitz's condition of order one with respect to $s$, i.e., there is a constant $\Delta$ such that for any $x, y \in(0,1)$

$$
\sup _{-\infty<t<\infty}\left|\phi_{1}(x, t)-\phi_{1}(y, t)\right| \leq \Delta|x-y| \text {. }
$$

(Note that the normal scores statistic does not satisfy this assumption).

We now introduce some notations. Let $S^{\circ}$ denote a r.v. $S$ centered at its expectation, i.e. $S^{\circ}=S-E S$. Denote

$$
\rho_{N j}=R_{N j}^{g} /(N+1), \quad \rho_{N j j}=E\left(\rho_{N j} \mid X_{j}\right) .
$$

Furthermore, we shall use the r.v. $\hat{S}_{N}$ (Hájek's projection), as an approximation of the statistic $T_{N}$, defined by

$$
\hat{S}_{N}=\sum_{\ell=1}^{N} E\left(S_{N} \mid X_{\ell}\right)-(N-1) E S_{N}
$$

where $S_{N}$ is the first two terms of Taylor's expansion of $T_{N}$ with approximate scores, i.e.,

$$
S_{N}=\sum_{j=1}^{N} c_{N j}\left\{\phi\left(\rho_{N j j}, X_{N j}\right)+\left(\rho_{N j}-\rho_{N j j}\right) \phi_{1}\left(\rho_{N j j}, X_{N j}\right)\right\} .
$$

Let $\Phi(\cdot)$ denote the standard normal c.d.f. and put $\hat{\sigma}_{N}^{2}=\operatorname{var} \hat{S}_{N}$. Then

THEOREM 2.1. Under assumptions (A), (B) and (C), uniformly in the region $0<x \leq \rho_{N} N^{1 / 6}, \quad \rho_{N}=o(1)$, we have as $N \rightarrow \infty$

$$
P\left(T_{N}-E T_{N}>\tau_{N} x\right)[1-\Phi(x)]^{-1}=1+o(1)
$$

which remains true if we replace $\tau_{N}$ by $\hat{\sigma}_{N}$.

REMARK 2.1. Note that the result of Kallenberg (1982) which deals with the statistic (1.4) and holds for the case of iid r.v.'s is a special case of our result, but we impose somewhat stronger assumption on the score generating function. This is due to our generalized statistic (1.1) and weaker assumptions on underlying distributions. However, for the case of iid r.v.'s, the results of the above theorem hold under relatively weaker assumptions (see Remark 4.1). 
From now on, we shall suppress the subscript $N$ in $c_{N j}, a_{N j}, R_{N j}^{g}, X_{N j}, \rho_{N j}$, $\rho_{N j j}$, etc., whenever it causes no confusion.

3. Preliminaries. In this section, we derive bounds on the $2 r$ th moments ( $r$ is any positive integer) for statistics defined in Lemma 3.1-3.5. These bounds play an important role in this paper and, since the method of their derivation depends heavily on that of Bickel (1974), we will give only the brief outline of the proofs.

Let $\left\{Y_{j}\right\}_{j=1}^{\infty}$ be a sequence of r.v.'s and $\left\{d_{j}\right\}_{j=1}^{\infty}$ a sequence of real numbers. Then we have the following lemmas.

LEMMA 3.1. Let $Z_{j}, j \geq 1$, be r.v.'s of the form $Z_{j}=g_{j}\left(Y_{1}, Y_{2}, \ldots, Y_{j}\right)$ such that for $j \geq 2, E\left(Z_{j} \mid Y_{1}, Y_{2}, \cdots, Y_{j-1}\right)=0$. If the sequence $\left\{d_{j}\right\}$ is nonincreasing in absolute values, then for any positive integers $r$ and $\ell$,

$$
E\left(\sum_{j=1}^{\ell} d_{j} Z_{j}\right)^{2 r} \leq(4 e)^{r}\left(\sum_{j=1}^{\ell} d_{j}^{2}\right)^{r} r^{r} \max _{i \leq j \leq \ell} E Z_{j}^{2 r} .
$$

LEMMA 3.2. Let $Y_{j}, j \geq 1$, be independent r.v.'s and let $\tilde{V}_{j k}$ be r.v.'s of the form $\tilde{V}_{j k}=g_{j k}\left(Y_{j}, Y_{k}\right), 1 \leq j, k<\infty$, such that for any $j$ and $k, j \neq k, E\left(\tilde{V}_{j k} \mid Y_{j}\right)$ $=E\left(\tilde{V}_{j k} \mid Y_{k}\right)=0$. Then, for any positive integers $\ell$ and $r$,

$$
E\left[(1 / \ell) \sum_{j=1}^{\ell} \sum_{k \neq j}^{\ell} d_{j} \tilde{V}_{j k}\right]^{2 r} \leq(4 e)^{2 r}\left(\sum_{j=1}^{\ell} d_{j}^{2}\right)^{r}(2 r)^{2 r} \ell^{-r} \max _{1 \leq j, k \leq \ell ; j \neq k} E \tilde{V}_{j k}^{2 r} .
$$

REMARK 3.1. Lemmas 3.1 and 3.2 are generalizations of Lemmas 1 and 4, respectively, of Hušková (1979). (We may point out that, in Hušková (1979), the proof of Lemma 4 is incorrect in its application of Lemma 1, especially in deriving (23) and (24) of her paper). Lemma 3.2 is also a generalization, as well as an improvement, of Lemma 2.2 of Bergström and Puri (1977).

Proof of Lemma 3.1. For $r \geq \ell$, the proof follows by applying Hölder's inequality to $\left(\sum_{j=1}^{\ell} d_{j} Z_{j}\right)^{2 r}$ and some routine computations. For $r \leq \ell$, the proof follows by induction on $\ell$ with $r$ fixed.

Proof of Lemma 3.2. Since the assumptions and the conclusion of this lemma are invariant under simultaneous permutation of $d_{j}$ 's and $Y_{j}^{\prime}$ 's, we may, without loss of generality, assume that $\left|d_{1}\right| \geq\left|d_{2}\right| \geq \cdots \geq\left|d_{\ell}\right|$.

Define $Z_{1}=\tilde{Z}_{1}=0, Z_{j}=\sum_{k=1}^{j-1} \tilde{V}_{j k}$ and $\tilde{Z}_{j}=\sum_{k=1}^{j-1} d_{k} \tilde{V}_{j k}, 2 \leq j \leq \ell$, so that $(1 / \ell) \sum_{j=1}^{\ell} \sum_{k \neq j}^{\ell} d_{j} \tilde{V}_{j k}=(1 / \ell)\left(\sum_{j=1}^{\ell} d_{j} Z_{j}+\sum_{j=1}^{\ell} \tilde{Z}_{j}\right)=\hat{V}_{\ell}$, say. The proof then follows by using the following facts:
(a) $E \hat{V}_{\ell}^{2 r} \leq \ell^{-2 r} 2^{2 r-1}(4 e)^{r} r^{r}\left\{\left(\sum_{j=1}^{\ell} d_{j}^{2}\right)^{r} \max _{1 \leq j \leq \ell} E Z_{j}^{2 r}+\ell^{r} \max _{1 \leq j \leq \ell} E \tilde{Z}_{j}^{2 r}\right\}$.
(b) $E Z_{j}^{2 r} \leq(4 e)^{r}(j-1)^{r} r^{r} \max _{1 \leq k \leq \ell ; k \neq j} E V_{j k}^{2 r} \quad$ and
(c) $E \tilde{Z}_{j}^{2 r} \leq(4 e)^{r}\left(\sum_{k=1}^{j-1} d_{k}^{2}\right)^{r} r^{r} \max _{1 \leq k \leq \ell ; k \neq j} E V_{k j}^{2 r}$.

Lemma 3.3. Let $T_{N}$ and $S_{N}$ be defined by (1.1) and (2.4). Then for any positive 
integer $r \geq 1, E\left(T_{N}^{0}-S_{N}^{0}\right)^{2 r} \leq(16 e \Delta)^{2 r}(2 r)^{2 r} N^{-r}$.

Proof. Denote $H_{j k}=u\left(X_{j}^{g}-X_{k}^{g}\right)-G_{k}\left(X_{j}^{g}\right), \quad 1 \leq j, k \leq N$. Then, we have

$$
\rho_{j}-\rho_{j j}=(N+1)^{-1} \sum_{k \neq j}^{N} H_{j k}
$$

and

$$
S_{N}=\sum_{j=1}^{N} c_{j}\left\{\phi\left(\rho_{j j}, X_{j}\right)+(N+1)^{-1} \sum_{k \neq j}^{N} H_{j k} \phi_{1}\left(\rho_{j j}, X_{j}\right)\right\} .
$$

First consider the statistic $T_{N}$ with approximate scores. Then, by Taylor's expansion, for some $0 \leq \lambda \leq 1$,

$$
T_{N}=\sum_{j=1}^{N} c_{j}\left\{\phi\left(\rho_{j j}, X_{j}\right)+\left(\rho_{j}-\rho_{j j}\right) \phi_{1}\left(\lambda \rho_{j j}+(1-\lambda) \rho_{j}, X_{j}\right)\right\} .
$$

Using (3.2) and (3.3) along with assumptions (B) and (C), we obtain

$$
E\left(T_{N}-S_{N}\right)^{2 r} \leq \Delta^{2 r} N^{r-1} \sum_{j=1}^{N} E\left(\rho_{j}-\rho_{j j}\right)^{4 r} .
$$

For each $j$, conditionally given $X_{j}^{g}, \rho_{j}-\rho_{j j}$ is the sum of independent r.v.'s with zero means and thus we may apply Lemma 3.1 to obtain that for any integer $r \geq 1$,

$$
E\left(\rho_{j}-\rho_{j j}\right)^{4 r} \leq(4 e)^{2 r}(2 r)^{2 r} N^{-2 r} .
$$

The proof follows using (3.4) and (3.5).

We now consider the exact scores. Let $\tilde{T}_{N}$ be the statistic (1.1) with exact scores and put for $1 \leq j \leq N, \tilde{a}_{j}(t)=E \phi\left(U_{N: j}, t\right)$ to distinguish it from the statistic $T_{N}$ with approximate scores. The proof then follows by using $\left|E \phi\left(U_{N: j}, t\right)-\phi(j /(N+1), t)\right| \leq \Delta N^{-1}, E\left(\tilde{T}_{N}^{0}-T_{N}^{0}\right)^{2 r} \leq(2 \Delta)^{2 r} N^{-r}$, $E\left(T_{N}^{0}-S_{N}^{0}\right)^{2 r} \leq 2^{2 r} E\left(T_{N}-S_{N}\right)^{2 r} \leq(8 e \Delta)^{2 r}(2 r)^{2 r} N^{-r}$ and routine computations.

LEMMA 3.4. Let $\hat{S}_{N}$ and $S_{N}$ be defined by (2.3) and (2.4). Then for any integer $r \geq 1, E\left(S_{N}^{0}-\hat{S}_{N}^{0}\right)^{2 r} \leq\left(8 e\left\|\phi_{1}\right\|\right)^{2 r}(2 r)^{2 r} N^{-r}$ where

$$
\left\|\phi_{1}\right\|=\sup _{0<s<1} \sup _{-\infty<t<\infty}\left|\phi_{1}(s, t)\right| \text {. }
$$

Proof. The proof follows by using Lemma 3.2 and noting that

$$
\begin{aligned}
E\left(S_{N} \mid X_{\ell}\right)= & c_{\ell} \phi\left(\rho_{\mathscr{C}}, X_{\ell}\right)+E S_{N}-c_{\ell} E \phi\left(\rho_{\mathscr{C}}, X_{\ell}\right) \\
& +(N+1)^{-1} \sum_{j=1}^{N} c_{j} E\left\{H_{j \ell} \phi_{1}\left(\rho_{j j}, X_{j}\right) \mid X_{\ell}\right\},
\end{aligned}
$$

and

$$
S_{N}^{0}-\hat{S}_{N}^{0}=(N+1)^{-1} \sum_{j=1}^{N} \sum_{k \neq j}^{N} c_{j}\left[H_{j k} \phi_{1}\left(\rho_{j j}, X_{j}\right)-E\left\{H_{j k} \phi_{1}\left(\rho_{j j}, X_{j}\right) \mid X_{k}\right\}\right]
$$

Lemma 3.5. Let $T_{N}$ and $\hat{S}_{N}$ be defined by (1.1) and (2.3). Then, for any real $r \geq 1 / 2$,

$$
E\left(T_{N}^{0}-\hat{S}_{N}^{0}\right)^{2 r} \leq\left(64 e\left(\Delta+\left\|\phi_{1}\right\|\right)\right)^{2 r}(2 r)^{2 r} N^{-r}
$$

Proof. Denote by $[x]$, the smallest integer $\geq x$. Then, applying Hölder's 
inequality,

$$
\left[E\left(T_{N}^{0}-\hat{S}_{N}^{0}\right)^{2 r}\right]^{[r] / r} \leq E\left(T_{N}^{0}-\hat{S}_{N}^{0}\right)^{2[r]} .
$$

Since, by the $C_{r}$-inequality,

the right-hand side term $\leq 2^{2[r]-1}\left[E\left(T_{N}^{0}-S_{N}^{0}\right)^{2[r]}+E\left(S_{N}^{0}-\hat{S}_{N}^{0}\right)^{2[r]}\right]$, we have

$$
\left[E\left(T_{N}^{0}-\hat{S}_{N}^{0}\right)^{2 r}\right]^{[r] / r} \leq 2^{2[r]-1}\left[E\left(T_{N}^{0}-S_{N}^{0}\right)^{2[r]}+E\left(S_{N}^{0}-\hat{S}_{N}^{0}\right)^{2[r]}\right] .
$$

Now using Lemmas 3.3 and 3.4 to the terms on the right-hand side, we obtain, after omitting some details of computations, that

$$
\left[E\left(T_{N}^{0}-\hat{S}_{N}^{0}\right)^{2 r}\right]^{[r] / r} \leq\left\{32 e\left(\Delta+\left\|\phi_{1}\right\|\right)\right\}^{2[r]}(2[r])^{2[r]} N^{-[r]} .
$$

Thus,

$E\left(T_{N}^{0}-\hat{S}_{N}^{0}\right)^{2 r}$

$$
\leq\left\{32([r] / r) e\left(\Delta+\left\|\phi_{1}\right\|\right)\right\}^{2 r}(2 r)^{2 r} N^{-r} \leq\left\{64 e\left(\Delta+\left\|\phi_{1}\right\|\right)\right\}^{2 r}(2 r)^{2 r} N^{-r}
$$

where the last inequality follows from the fact that $1 \leq[r] / r \leq 2$ for any real $r \geq 1 / 2$.

4. Proof of Theorem 2.1. By assumption (A) and Lemma 3.5, we have

$$
\begin{aligned}
\left|\hat{\sigma}_{N}^{2}-\tau_{N}^{2}\right| & =\left|2 \operatorname{Cov}\left(T_{N}, \hat{S}_{N}-T_{N}\right)+\operatorname{Var}\left(\hat{S}_{N}-T_{N}\right)\right| \\
& \leq 2 \tau_{N}\left(\operatorname{Var}\left(\hat{S}_{N}-T_{N}\right)\right)^{1 / 2}+\operatorname{Var}\left(\hat{S}_{N}-T_{N}\right)
\end{aligned}
$$

which implies that $\hat{\sigma}_{N}^{2} / \tau_{N}^{2}=1+O\left(N^{-1 / 2}\right)$ and that there is a positive constant $\sigma$ such that for all $N \geq N_{0}$,

$$
\hat{\sigma}_{N}^{2} \geq \sigma^{2} .
$$

Hence, in order to prove Theorem 2.1, it is sufficient to show that

$$
P\left(T_{N}-E T_{N}>\hat{\sigma}_{N} x\right)[1-\Phi(x)]^{-1}=1+o(1)-
$$

uniformly in the region $0<x<\rho_{N} N^{1 / 6}$.

By standard arguments we have

$$
\begin{aligned}
& P\left(T_{N}^{0}>\hat{\sigma}_{N} x\right) \leq P\left(\hat{S}_{N}^{0}>\left(x-N^{-1 / 6}\right) \hat{\sigma}_{N}\right)+P\left(\left|T_{N}^{0}-\hat{S}_{N}^{0}\right|>N^{-1 / 6} \hat{\sigma}_{N}\right), \\
& P\left(T_{N}^{0}>\hat{\sigma}_{N} x\right)>P\left(\hat{S}_{N}^{0}>\left(x+N^{-1 / 6}\right) \hat{\sigma}_{N}\right)-P\left(\left|T_{N}^{0}-\hat{S}_{N}^{0}\right|>N^{-1 / 6} \hat{\sigma}_{N}\right) .
\end{aligned}
$$

Using Chebyshev's inequality and applying Lemma 3.5 with $r=1 / 2 \delta N^{1 / 3}$, $\delta=\sigma\left(64\left(\Delta+\left\|\phi_{1}\right\|\right) e^{2}\right)^{-1}$, we get

$$
P\left(\left|T_{N}^{0}-\hat{S}_{N}^{0}\right|>N^{-1 / 6} \hat{\sigma}_{N}\right) \leq E\left(T_{N}^{0}-\hat{S}_{N}^{0}\right)^{2 r} N^{r / 3} \hat{\sigma}_{N}^{-2 r} \leq \exp \left(-\delta N^{1 / 3}\right)
$$

which implies that uniformly for $0<x \leq \rho_{N} N^{1 / 6}$,

$$
\begin{aligned}
P\left(\left|T_{N}^{0}-\hat{S}_{N}^{0}\right|>N^{-1 / 6} \hat{\sigma}_{N}\right)[1 & -\Phi(x)]^{-1} \\
& \leq \exp \left(-\delta N^{1 / 3}\right)\left[1-\Phi\left(\rho_{N} N^{1 / 6}\right)\right]^{-1}=o(1)
\end{aligned}
$$

where the last equality follows by Lemma VII.1.2 of Feller (1968). 
Now, in view of (4.3) and (4.5), to prove (4.2) it suffices to show that as $N \rightarrow \infty$

$$
P\left(\hat{S}_{N}^{0}>x_{N} \hat{\sigma}_{N}\right)[1-\Phi(x)]^{-1}=1+o(1)
$$

uniformly in $\left|x_{N}-x\right|=N^{-1 / 6}, 0 \leq x \leq \rho_{N} N^{1 / 6}$.

Using (2.3) and (3.6) we can write $\hat{S}_{N}^{0}=\sum_{j=1}^{N} \hat{S}_{N}^{(j)}$ where

$$
\begin{aligned}
\hat{S}_{N}^{(j)}= & c_{j}\left[\phi\left(\rho_{j j}, X_{j}\right)-E \phi\left(\rho_{j j}, X_{j}\right)\right] \\
& +(N+1)^{-1} \sum_{k \neq j}^{N} c_{k} E\left\{\left[u\left(X_{k}^{g}-X_{j}^{g}\right)-G_{j}\left(X_{k}^{g}\right)\right] \phi_{1}\left(\rho_{k k}, X_{k}\right) \mid X_{j}\right\} .
\end{aligned}
$$

Thus $\hat{S}_{N}^{0}$ is a sum of independent r.v.'s with means zero and

$$
\begin{gathered}
\left|S_{N}^{(j)}\right| \leq 2\left|c_{j}\right|\|\phi\|+(N+1)^{-1} \sum_{k \neq j}^{N}\left|c_{k}\right|\left\|\phi_{1}\right\| \\
\leq\left(2\|\phi\|+\left\|\phi_{1}\right\|\right) A_{1} N^{-1 / 3}, \quad 1 \leq j \leq N,
\end{gathered}
$$

where $\|\phi\|=\sup _{0<s<1} \sup _{-\infty<t<\infty}|\phi(s, t)|$. Furthermore, there is an integer $N_{0}$ such that for all $N \geq N_{0}$ and $0<x<\rho_{N} N^{1 / 6},\left|x_{N}-x\right| \leq N^{-1 / 6}$,

$$
0<\left(2\|\phi\|+\left\|\phi_{1}\right\|\right) A_{1} N^{-1 / 3} \hat{\sigma}_{N}^{-1} x_{N} \leq 1 / 12
$$

in view of (4.1). Thus we can use Theorem 1 of Feller (1943) (cf. also Petrov, 1975 , page 253) to obtain that for all $N \geq N_{0}$,

(4.8) $P\left(\hat{S}_{N}^{0}>x_{N} \hat{\sigma}_{N}\right)=\exp \left\{-1 / 2 x_{N}^{2} Q_{N}\left(x_{N}\right)\right\}\left[1-\Phi\left(x_{N}\right)+\theta_{N} \lambda_{N} \exp \left(-1 / 2 x_{N}^{2}\right)\right]$

where

$$
\begin{gathered}
\lambda_{N}=\left(2\|\phi\|+\left\|\phi_{1}\right\|\right) A_{1} N^{-1 / 3} \hat{\sigma}_{N}^{-1}, \quad \theta_{N}<7.465, \\
Q_{N}(x)=\sum_{j=1}^{\infty} q_{N j} x^{j}, \quad q_{N 1}=3^{-1} \hat{\sigma}_{N}^{-3} \sum_{j=1}^{N} E\left(\hat{S}_{N}^{(j)}\right)^{3}, \\
\left|q_{N j}\right|<8^{-1}\left(12 \lambda_{N}\right)^{j}, \quad j \geq 2 .
\end{gathered}
$$

Note that $\left|x_{N}\right| \leq \rho_{N} N^{1 / 6}+N^{-1 / 6}$. Since $\rho_{N}=o(1)$ we have $x_{N}=o\left(N^{1 / 6}\right)$ as $N \rightarrow \infty$. Let $K>0$ be such that

$$
\left|x_{N}\right| \leq \frac{K \hat{\sigma}_{N} N^{1 / 6}}{12 A_{1}\left(2\|\phi\|+\left\|\phi_{1}\right\|\right)}, \quad N \geq 1 .
$$

Also it follows by assumption (B) and (4.1) that

$$
\left|q_{N 1} x_{N}\right| \leq \hat{\sigma}_{N}^{-3} 4 / 3\left(2\|\phi\|^{3}+\left\|\phi_{1}\right\|^{3}\right) \sum_{j=1}^{N}\left|c_{j}\right|^{3} x_{N} \leq A_{3} N^{-1 / 3}
$$

where $A_{3}$ is an absolute constant. Hence combining (4.9), (4.10) and (4.11) we obtain that

$$
\left|Q_{N}\left(x_{N}\right)\right| \leq A_{3} N^{-1 / 3}+\sum_{j=2}^{\infty}\left|q_{N j} x_{N}^{j}\right| \leq A_{3} N^{-1 / 3}+8^{-1} \sum_{j=2}^{\infty}\left(K N^{-1 / 6}\right)^{j}
$$

which implies that as $N \rightarrow \infty$.

$$
x_{N}^{2} Q_{N}\left(x_{N}\right)=x_{N}^{2} O\left(N^{-1 / 3}\right)=o\left(N^{1 / 3}\right) O\left(N^{-1 / 3}\right)=o(1)
$$

uniformly in $0<x \leq \rho_{N} N^{1 / 6}$. Moreover Lemma VII.1.2 of Feller (1968) ensures 
that

$$
\begin{gathered}
\theta_{N} \lambda_{N} \exp \left(-1 / 2 x_{N}^{2}\right)\left[1-\Phi\left(x_{N}\right)\right]^{-1}=O\left(\lambda_{N} x_{N}\right)=o(1), \\
{\left[1-\Phi\left(x_{N}\right)\right]^{-1}[1-\Phi(x)]=1+o(1) .}
\end{gathered}
$$

(4.2) now follows uniformly in $0<x \leq \rho_{N} N^{1 / 6}$ by using (4.8), (4.12) and (4.13).

REMARK 4.1. The above theorem obviously holds for the case of the iid r.v.'s. However, for such a case, and for the statistics (1.4) and (1.5), the theorem holds under somewhat weaker assumptions on the score generating function, if we assume somewhat stronger assumption on the underlying distribution function. For the case of the statistic (1.4), we refer to Kallenberg (1982). For the case of the statistic (1.5), we have the following theorem:

THEOREM 4.1. Let $X_{N j}, 1 \leq j \leq N, N \geq 1$ be iid r.v.'s with a continuous c.d.f. $F_{N}(x)$ symmetric about 0 . Assume the following:

Assumption (D). $\quad \sum_{j=1}^{N} c_{N j}^{2}=1, \max _{1 \leq j \leq N}\left|c_{N j}\right| \leq A_{1} N^{-1 / 3}$ where $A_{1}$ is an absolute constant.

Assumption (E). The score generating function $\tilde{\phi}$ (defined in Section 1) is not identically zero and satisfies a Lipschitz condition of order 1 on $(0,1)$, that is, there exists a constant $C$ such that $|\tilde{\phi}(t)-\tilde{\phi}(s)| \leq C|t-s|$ for all $t, s \in(0,1)$.

Then, the conclusion of the Theorem 2.1 holds.

To prove this theorem we use the following two lemmas and proceed essentially as in the proof of Theorem 2.1. (As before, we suppress the subscript $N$ in $c_{N j}$, $X_{N j}$ etc. whenever it causes no confusion).

LeMma 4.1. Let $Z_{1}, \cdots, Z_{N}$ be random variables such that for any permutation $\left(i_{1}, \cdots, i_{N}\right)$ of $(1, \cdots, N)$

$$
E \prod_{j=1}^{N} Z_{j}^{\alpha_{j}}=E \prod_{j=1}^{N} Z_{i_{j}}^{\alpha_{j}}
$$

where $\alpha_{j}$ 's are nonnegative integers such that $\sum_{j=1}^{N} \alpha_{j}=2 k, k \geq 1$ integer. Furthermore, assume that

$$
E \prod_{j=1}^{N} Z_{j}^{\alpha_{j}}=0
$$

if at least one of the $\alpha_{j}$ 's is odd. Then if $\sum_{j=1}^{N} c_{j}^{2}=1$, for any integer $k \leq$ $d\left(\max _{1 \leq j \leq N}\left|c_{j}\right|\right)^{-1}, d>0$

$$
E\left(\sum_{j=1}^{N} c_{j} Z_{j}\right)^{2 k} \leq 2^{2 k+1}(\max \{1, d\})^{2 k} k^{k} E Z_{1}^{2 k} .
$$

Consider now the statistic $S_{N}=\sum_{j=1}^{N} c_{j} \tilde{\phi}\left(F_{N}^{*}\left(\left|X_{j}\right|\right)\right) \operatorname{sgn} X_{j}$ where $F_{N}^{*}(x)=$ $P\left(\left|X_{1}\right| \leq x\right), 0 \leq x<\infty$. 
LEMMA 4.2. For all real $p, 1 \leq p \leq N$,

$$
E\left(T_{N}-S_{N}\right)^{2 p} \leq A_{2}^{2 p}(2 p)^{2 p} N^{-p}\left(\max \left\{1, p \max _{1 \leq j \leq N}\left|c_{j}\right|\right\}\right)^{2 p}
$$

where $A_{2}$ is a constant independent of $N$ and $p$.

Proof of Lemma 4.1. Because of (4.14) and (4.15), the multinomial expansion yields

$$
\begin{aligned}
E\left(\sum_{j=1}^{N} c_{j} Z_{j}\right)^{2 k} & \\
= & \sum_{\alpha=1}^{k} \sum_{\left(k_{1}, \cdots, k_{\alpha}\right) \in A_{\alpha}} \frac{(2 k) !}{\prod_{\nu=1}^{\alpha}[(2 \nu) !]^{k_{\nu}} \prod_{\nu=1}^{\alpha}\left(k_{\nu}\right) !} \\
& \cdot \sum_{\left(i_{1}, \cdots, i_{K_{\alpha}}\right) \neq} E \prod_{j=1}^{K_{1}}\left(c_{i_{j}} Z_{i_{j}}\right)^{2} \prod_{j=K_{1}+1}^{K_{2}}\left(c_{i_{j}} Z_{i_{j}}\right)^{4} \cdots \prod_{j=K_{\alpha-1}}^{K_{\alpha}}\left(c_{i_{j}} Z_{i_{j}}\right)^{2 \alpha}
\end{aligned}
$$

where $k_{j}, 1 \leq j \leq \alpha$ are nonnegative integers, $K_{\beta}=\sum_{\nu=1}^{\beta} k_{\nu}, 1 \leq \beta \leq \alpha, A_{\alpha}=$ $\left\{\left(k_{1}, k_{2}, \cdots, k_{\alpha}\right): \sum_{\nu=1}^{\alpha} \nu k_{\nu}=k\right\}$ and $\sum_{\left(i_{1}, \cdots, i_{K_{\alpha}}\right) \neq}$ means that the sum is taken over mutually different indices $1 \leq i_{1}, i_{2}, \cdots i_{K_{\alpha}} \leq N$.

From (4.14) and generalized Hölder's inequality, it follows that

$$
\begin{aligned}
\sum_{\left(i_{1}, \cdots, i_{K_{\alpha}}\right) \neq} E \prod_{j=1}^{K_{1}}\left(c_{i_{j}} Z_{i_{j}}\right)^{2} \prod_{j=K_{1}+1}^{K_{2}}\left(c_{i_{j}} Z_{i_{j}}\right)^{4} \cdots \prod_{j=K_{\alpha-1}}^{K_{\alpha}}\left(c_{i_{j}} Z_{i_{j}}\right)^{2 \alpha} \\
\leq \sum_{\left(i_{1}, \cdots, i_{K_{\alpha}}\right)} \neq \pi\left(k_{1}, k_{2}, \cdots, k_{\alpha}\right) E Z_{1}^{2 k}
\end{aligned}
$$

where

$$
\pi\left(k_{1}, k_{2}, \cdots, k_{\alpha}\right)=\prod_{j=1}^{K_{1}} c_{i_{j}}^{2} \prod_{j=K_{1}+1}^{K_{2}} c_{i_{j}}^{4} \cdots \prod_{j=K_{\alpha-1}+1}^{K_{\alpha}} c_{i_{j}}^{2 \alpha} .
$$

Now, if we let $p_{1}=\cdots=p_{K_{1}}=2$,

$$
p_{K_{1}+1}=\cdots=p_{K_{2}}=4, \cdots, p_{K_{\alpha-1}+1}=\cdots=p_{K_{\alpha}}=2 \alpha,
$$

by using the conditions $k \leq d\left(\max _{1 \leq j \leq N}\left|c_{j}\right|\right)^{-1}$ and $\sum_{j=1}^{N} c_{j}^{2}=1$; we get

$$
\begin{aligned}
\sum_{\left(i_{1}, \cdots, i_{k_{\alpha}}\right) \neq \pi}\left(k_{1}, \cdots, k_{\alpha}\right) & \leq \sum_{\rho_{1}, \cdots, \ell_{K_{\alpha}}=1}^{N} c_{c_{\ell_{1}}}^{p_{1}} \cdots c_{\ell_{K_{\alpha}}}^{p_{K_{\alpha}}} \leq \prod_{j=1}^{K_{\alpha}}\left(\sum_{i=1}^{N} c_{i}^{p_{j}}\right) \\
& \leq\left(\max \left|c_{i}\right|\right)^{2 k-2 K_{\alpha}} \leq(d / k)^{2 k-2 K_{\alpha}} .
\end{aligned}
$$

Thus, relations (4.17)-(4.19) yield the estimate

$$
\begin{aligned}
& E\left(\sum_{j=1}^{N} c_{j} Z_{j}\right)^{2 k} \\
& \quad \leq \sum_{\alpha=1}^{k} \sum_{\left(k_{1}, \cdots, k_{\alpha}\right) \in A_{\alpha}} \frac{(2 k) !}{\prod_{\nu=1}^{\alpha}[(2 \nu) !]^{k_{\nu}} \prod_{\nu=1}^{\alpha}\left(k_{\nu}\right) !}\left(\frac{d}{k}\right)^{2 k-2 K_{\alpha}} E Z_{1}^{2 k} .
\end{aligned}
$$

Further, by Stirling's formula (see Feller, 1968)

$$
(2 \pi)^{1 / 2} n^{n+1 / 2} e^{-n}<n ! \leq(2 \pi)^{1 / 2} n^{n+1 / 2} e^{-n}(1+(1 / 4 n))
$$

we obtain, after some computations, that

$$
\frac{\prod_{\nu=1}^{\alpha}(\nu !)^{k_{\nu}}(2 k) !\left(k-K_{\alpha}\right) !}{\prod_{\nu=1}^{\alpha}[(2 \nu) !]^{k_{\nu}}(k !)^{2}}\left(\frac{d}{k}\right)^{2 k-2 K_{\alpha}} \leq 2^{2 k+1}(\max \{1, d\})^{2 k} .
$$


Finally, on the basis of the expansion

$$
k^{k}=\sum_{\alpha=1}^{k} \sum_{\left(k_{1}, \cdots, k_{\alpha}\right) \in A_{\alpha}}(k !)^{2} /\left\{\prod_{\nu=1}^{\alpha}(\nu !)^{k_{\nu}} \prod_{\nu=1}^{\alpha}\left(k_{\nu}\right) !\left(k-K_{\alpha}\right) !\right\}
$$

inequalities (4.20) and (4.21) lead to the assertion (4.16) in Lemma 4.1.

Proof of Lemma 4.2. Let $1 \leq p \leq N$ be any given integer and let $Z_{j}=$ $\left\{\tilde{\phi}\left(R_{j}^{+} /(N+1)\right)-\tilde{\phi}\left(F^{*}\left(\left|X_{j}\right|\right)\right)\right\}$ sgn $X_{j}, \quad 1 \leq j \leq N$. Then, using Lemma 4.1 with $d=p \max _{1 \leq j \leq N}\left|c_{j}\right|$, it follows that

$$
E\left(T_{N}-S_{N}\right)^{2 p} \leq 2^{2 p+1}\left(\max \left\{1, p \max _{1 \leq j \leq N}\left|c_{j}\right|\right\}\right)^{2 p} p^{p} E Z_{1}^{2 p} .
$$

Now, using Hölder's inequality and proceeding as in Kallenberg (1982), we obtain the desired result for any real $p, \quad 1 \leq p \leq N$. The details are omitted.

\section{REFERENCES}

Bergström, H. and Puri, M. L. (1977). Convergence and remainder terms in linear rank statistic. Ann. Statist. 5 671-680.

BICKEL, P. J. (1974). Edgeworth expansions in nonparametric statistics. Ann. Statist. 2 1-20.

BJERVE, S. (1977). Error bounds for linear combinations of order statistics. Ann. Statist. 5 357-369.

Cramér, H. (1938). Sur un nouveau theoreme-limite de la theorie des probabilities. Actualites Sci. Ind. 736 5-23.

Does, R. J. M. M. (1982). Higher Order Asymptotics for Simple Linear Rank Statistics. Mathematisch Centrum, Amsterdam.

FELleR, W. (1943). Generalization of a probability limit theorem of Cramér. Trans. Amer. Math. Soc. 54 361-372.

Feller, W. (1968). An Introduction to Probability Theorem and its Applications, Vol. I, 3rd edition. Wiley, New York.

HÁJEK, J. (1962). Asymptotically most powerful rank order tests. Ann. Math. Statist. 33 1129-1147.

HÁJEK, J. (1968). Asymptotic normality of simple linear rank statistics under alternatives. Ann. Math. Statist. 39 325-346.

Helmers, R. (1977). The order of the normal approximation for linear combinations of order statistics with smooth weight functions. Ann. Probab. 5 940-953.

Helmers, R. (1980). A Berry-Esséen theorem for linear combinations of order statistics with smooth weight functions. Ann. Statist. 8 1361-1374.

Helmers, R. (1981). A Berry-Esseen theorem for linear combinations of order statistics. Ann. Probab. 9 342-347.

Ho, S. T. and Chen, L. Y. H. (1978). An $L_{p}$ bound for the remainder in a combinatorial central limit theorem. Ann. Probab. 6 231-249.

Hoeffding, W. (1951). A combinatorial central limit theorem. Ann. Math. Statist. 22 558-566.

HuŠKovÁ, M. (1970). Asymptotic distribution of simple linear rank statistics for testing symmetry. Z. Wahrsch. verw. Gebiete. 14 308-322.

HuŠKovÁ, M. (1977). The rate of convergence of simple linear rank statistics under hypothesis and alternatives. Ann. Statist. 5 658-670.

HušKovÁ, M. (1979). The rate of convergence of simple linear rank statistics under alternatives. Contributions to Statistics, 99-108. Ed. by J. Jurečková. Academic Publishing House, Prague.

JUREČKovÁ, J. AND PURI, MADAN L. (1975). Order of normal approximation for rank test statistics distribution. Ann. Probab. 3 526-533.

KallenberG, W. C. M. (1982). Cramér type large deviations for simple linear rank statistics. $Z$. Wahrsch. verw. Gebiete. 60 403-409.

Malevich, T. L. and AbDalimov, B. (1979). Large deviation probabilities for $U$-statistics. Theory Probab. Appl. 24 215-219. 
Moтоo, M. (1956). On the Hoeffding's combinatorial central limit theorem. Ann. Inst. Statist. Math. 8 145-154.

Petrov, V. V. (1975). Sums of Independent Random Variables. Springer-Verlag, Berlin.

PuRI, M. L. and RAlescu, S. (1984). Centering of signed rank statistics with continuous score generating function. Teor. Veroyatnost. $i$ Primenen. 29 (to appear).

PURI, M. L. and SEOH, M. (1984a). Berry-Esséen theorems for signed linear rank statistics with regression constants. Colloquium on Limit Theorems in Probability and Statistics. Veszprém (Hungary). To appear.

PURI, M. L. and SEOH, M. (1984b). Edgeworth expansions for signed linear rank statistics with regression constants. J. Statist. Plann. Inference 10 137-149.

PURI, M. L. and SEOH, M. (1984c). Edgeworth expansions for signed linear rank statistics under near location alternatives. J. Statist. Plann. Inference 10 289-309.

PURI, M. L. and SEOH, M. (1984d). On the rate of convergence in normal approximation for a class of statistics. Tech. report no. 23, Dept. of Mathematics, Indiana University, Bloomington.

PURI, M. L. and WU, T. J. (1985). The order of normal approximation for signed linear rank statistics. Teor. Verojatnost. i Primenen. (To appear).

Robinson, J. (1977). Large deviation probabilities for samples from a finite population. Ann. Probab. 5 913-925.

SHORACK, G. R. (1969). Asymptotic normality of linear combinations of functions of order statistics. Ann. Math. Statist. 40 2041-2050.

ShoraCK, G. R. (1972). Functions of order statistics. Ann. Math. Statist. 43 412-427.

STIGLER, S. M. (1974). Linear functions of order statistics with smooth weight functions. Ann. Statist. 2 676-693.

VON BAHR, B. (1976). Remainder term estimate in a combinatorial limit theorem. Z. Wahrsch. verw. Gebiete 35 131-139.

\author{
Munsup SeOH \\ DEPARTMENT OF MATHEMATICS \\ AND STATISTICS \\ WRIGHT STATE UNIVERSITY \\ DAYTON, OHIO 45435
}

\author{
STEFAN S. RALESCU \\ Division of APPlied Mathematics \\ BROWN UNIVERSITY \\ ProvidenCE, R.I. 02912
}

\author{
MADAN L. PURI \\ DEPARTMENT OF MATHEMATICS \\ INDIANA UNIVERSITY \\ BLOOMINGTON, INDIANA 47405
}

